\title{
SÉMIOTIQUE DE L'ÉVÉNEMENT \\ DE "L'ÉTRANGE VOYAGE DE M. DALDRY" DE MARC LÉVY.
}

Dr. Amany M. Magdy ${ }^{(*)}$

Notre investigation s'intéresse à analyser la sémiotique de l'événement dans l'œuvre de MARC LÉVY, intitulé "L'étrange voyage de M.Daldry. Notre problématique tentera répondre à la question suivante: dans quelle mesure les circonstances,et la société peuvent déterminer la formation de l'identité d'un sujet? Et comment rendre le négatif, positif? Comment se créer une identité unique en tournant le noir en blanc? Comment peut-on opérer sa propre catharsis en s'appuyant sur l'espace et les actions qui s'y déroulent?

La sémiotique de l'événement selon Bernard LAMIZET, s'intéresse à analyser les conséquences des événements,leurs temps, l'espace de leurs déroulements sur la formation de l'identité d'un sujet donné. Le théoricien fait la différence entre deux genres de sémiotique : «celle de l'évènement, qui est une sémiotique de l'espace et du temps, et celle de l'identité , qui est une sémiotique de la médiation entre sa dimension singulière et sa dimension collective:

La sémiotique à dimension particulière met en relief les interactions entre le temps,l'espace de l'événement et leurs conséquences sur la formation de l'identité de la personne. la sémiotique à dimension collective s'intéresse à analyser toutes ces interactions et leurs conséquences sur une société donnée.

L'analyse sémiotique de la vie des deux sujets actants, héros de notre corpus pour découvrir jusqu'à quel point les Événements dans une société donnée, dans une époque donnée peuvent contribuer à la formation de leurs identités.

${ }^{(*)}$ Département de Langue française - Faculté Al Alsun - Université de Minia 


\section{INTRODUCTION:}

Marc Lévy est un écrivain français contemporain, traduit en 49 langues, dont les romans se vendent par millions et demeurent à la tête des bestsellers pour de longues durées. Quoique parisien, il vit à New York, estime beaucoup le voyage,qui de son point de vue, enrichit sa vision du monde. Meticuleux et appliqué, il préfère se qualifier d'artisan et non pas d'écrivain grâce au plaisir qu'il se donne en écrivant. A l'âge de 17 ans, il travaille comme secouriste à la Croix Rouge, ce qui aiguise chez lui le sens que tout ce qui touche à l'humanité est précieux. Ses origines juives et la participation de son père à la résistance le dotent d'une certaine profondeur. Il fonde un cabinet d'architecture,puis d'informatique, mais ne se consacre exclusivement à l'écriture qu'après le succès éclatant de son premier roman "Et si c'était vrai" publié en l'an 2000.

Notre corpus intitulé "L'étrange voyage de M.Daldry" publié en 2011 est son onzième roman. Lévy a voulu rendre hommage aux charmes d'Istanbul en tant qu'un lieu riche d'histoires, de couleurs et de parfums.

Les romans de Marc Lévy demeurent pour une longue période à la tête des bestsellers grâce à son esprit innovant . Ses romans procurent une sensation de bonheur au lecteur. Il sait comment propager un certain sens de bonheur subtil au fil de ses pages grâce à sa touche humaniste.Une délectation magique se dégage de ses romans grâce à cette sensibilité.

"L'étrange voyage de M. Daldry" est un roman à la fois simple et complexe. Le thème du roman reflète l'amour de Marc Lévy pour le voyage. Le roman relate la vie solitaire de deux sujets qui se cotôient sur le même palier d'une ancienne maison victorienne. La jeune femme, orpheline depuis la deuxième guerre mondiale,vit seule après la mort de ses parents.Elle est une créatrice de parfums talentueuse,mais solitaire. 
Sur le même palier,réside $M$. Daldry, un peintre et un musicien à la fois. Un homme calme, enfermé qui a horreur de la vivacité bruyante de sa voisine ${ }^{1}$.

$\mathrm{Au}$ début de l'histoire,ils entretenaient des relations houleuses, mais la solitude aidant, les deux voisins sympathisent. Ils concluent un accord étrange de voyager en Orient pour fuir la grisaille londonienne, jouir du soleil oriental, et chercher de nouvelles compositions florales de parfums ${ }^{2}$.

L'histoire relate l'accomplissement d'un accord pragmatique de profits mutuels. Alice accepte de laisser son appartement à verrière éclairée à son voisin qui en a besoin pour sa peinture. Lui, il financera leur voyage en Orient et sera remboursé lorsque Alice réussira à composer et à vendre le parfum inspiré des lieux visités. Ici, Marc Levy a voulu confronter ses personnages aux situations dramatiques de leurs vies respectives. Il met en place deux protagonistes vivant chacun à part, d'un drame respectif et essaye de montrer comment ils vont surmonter leurs drames personnels.

Notre problématique vise à étudier la sémiotique de l'événement en tant qu' interaction complexe entre les événements qui se déroulent dans la vie des sujets actants, la fonction de l'espace et l'impact de ces circonstances enchevêtrées sur la formation de l'identité des actants. sa sensibilité fine apparaît dans le choix des métiers de ses protagonistes: un peintre qui joue au piano et une compositrice de parfums.

La sémiotique de l'événement s'intéresse essentiellement à la signification subtile d'un événement donné et sa réception

${ }^{1}$ permettez nous de souligner la finesse du choix des métiers des deux protagonistes. Marc Levy est sensible à la musique et joue au piano. Il apprécie également les qualités précieuses de la parfumerie. La sensibilité humaine détermine ses choix.

${ }^{2}$ Notons l'amour de Marc Levy pour le voyage qui nourrit et enrichit la créativité de l'écrivain. 
spéciale par un sujet donné. C'est à dire que la réception de l'événement et son impact diffèrent totalement d'une personne à l'autre; chacune selon son milieu, son histoire personnelle, son passé et sa capacité psychique.

Nous nous basons sur la sémiotique de l'événement Lamizet. Elle nous est parue la plus pertinente à analyser le cas des sujets actants présentés par Marc Lévy. Nous avons également eu recours à la sociologie de Pierre Bourdieu pour approfondir l'analyse de la formation de l'identité chez le sujet actant Alice. En ce qui concerne le second sujet, la psychanalyse nous a aidé à comprendre ses difficultés psychiques.

Notre travail se divise en 3 points essentiels: la sémiotique de l'événement, la sémiotique de l'espace et la sémiotique de l'identité. Nous nous intéressons à l'enchevêtrement entre ces trois axes pour démontrer l'impact des événements sur la formation de l'identité des personnages.

\section{I-Sémiotique de l'événement}

Bernard Lamizet définit la sémiotique de l'événement comme suit:

"Le concept d'événements,étymologiquement,renvoie à la spatialité : en effet, il se fonde sur l'articulation de l'intériorité et de l'extériorité.(...) Le terme désigne donc un fait qui vient du dehors et qui entre à l'intérieur - à l'intérieur de l'espace de communication, de l'espace spéculaire". ${ }^{3}$

Lamizet explique que chaque événement construit son sens à travers son interaction entre plusieurs axes: l'espace où se déroule l'événement,le temps de l'événement et son impact sur la destinée des personnages. Cet événement ne peut acquérir un

\footnotetext{
${ }^{3}$ Lamizet,Bernard,La sémiotique de l'événement: une sémiotique de l'espace et du temps.2011<.halshs,- 00604452>p. 5. https// halshs.archives-ouvertes.fr/halshs-00604452.
} 
sens particulier, une signification particulière qu'après l'étude de ces trois points. C'est ainsi qu'un fait comme la persécution des Arméniens en Turquie en 1915 ne devient un événement qu'à partir du moment où les médias,en la racontant et en la décrivant,lui donnent une signification particulière pour montrer son impact sur la vie des personnages. Il y a donc une médiation entre la signification politique et historique de l'événement et son interférence dans la vie personnelle des sujets.

Nous allons démontrer comment la logique des événements historiques: massacre des Arméniens aide- t-elle à fonder l'identité d'Alice. Notre héroïne est confrontée ,à plusieurs reprises, aux événements historiques. L'analyse essayera de démontrer comment son identité à été formée à la lueur de cette confrontation.

Les grands événements historiques présentés par Marc Lévy dans le corpus sont:les massacres perpétrés par les Turques contre les Arméniens en 1915 et la Deuxième Guerre Mondiale.

\section{Massacre des Arméniens en Turquie:}

Alice ou Anouche est un sujet arménien. Elle a mené une vie heureuse et paisible jusqu'à l'âge de cinq ans entre sa famille à Istanbul. Son père cordonnier à été tué par la police turque pendant le massacre des Arméniens. Ceux-ci ont été soupçonnés de comploter avec les Russes contre la Turquie. Du jour au lendemain, cet enfant se trouve arraché de sa famille. Sa mère est persécutée,elle aussi, pendant le massacre. La petite fille et son frère furent confiés à leurs nourrices. Pour sauver la vie des enfants, la nourrice, Mme Yalmaz, entreprend un long voyage nocturne afin d'atteindre son domicile. Les périples de ce voyage étaient terrifiants pour les deux enfants.

Le lendemain de la persécution, Anouche devient amnésique; elle perd également sa capacité oratoire. Incapable 
d'investir cet événement atroce, son inconscient fragile demeure bloqué.

Pour la soigner, Mme Yalmaz la conduit chez un couple pharmacien qui fabrique des traitements avec des herbes médicinales. Le couple pharmacien était des chercheurs britanniques, résidant en Turquie pour effectuer des recherches pharmacologiques à l'université d'Istanbul. Ils adoptent la jeune fille:

"(Ils) tenaient une sorte de dispensaire clandestin. Dès qu'ils rentraient de l'hôpital universitaire,votre mère pratiquait les soins et faisait les pansements tandis que votre père distribuait les médicaments qu'il avait pu trouver et les remèdes médicinaux qu'il préparait lui-même". ${ }^{4}$

Au sein de cette nouvelle famille, la jeune fille commence à faire des progrès. Elle récupère la capacité à communiquer oralement.

\section{Deuxième GUERRE Mondiale:}

Avec la déclaration de la Deuxième Guerre Mondiale, La Turquie s'allie à l'Allemagne. Les parents adoptifs d'Anouche se trouvent du jour au lendemain des sujets britanniques sur une terre ennemie. Ils se sont trouvés dans l'obligation de fuir la Turquie de peur de tomber proie à des vengeances probables.

$\mathrm{Au}$ lieu de suivre ses traitements sur sa terre natale pour reprendre conscience de sa vie passée, Anouche se trouve transportée à Londres. Ses parents adoptifs ont eu soin de taire la réalité traumatique à la jeune fille de peur de la perturber davantage. Ils la renomment Alice, lui fournissent une vie calme et heureuse jusqu'à leur disparition soudaine sous les bombardements allemands pendant la Deuxième Guerre Mondiale à Londres.

Il semble que les événements politiques ont eu raison de la vie psychique de la jeune fille. Ils ont affecté son inconscient

${ }^{4}$ Lévy (Marc), L'étrange voyage de Mr. Dalry,Paris,Robert Laffont,2011, P.212. 
fragile. Si la perte de la fonction oratoire et la perte de la mémoire ont été des conséquences au premier événement traumatique,la mort brutale de ses parents adoptifs a achevé la catastrophe. Alice se trouve totalement bloquée, elle n'arrive même pas à investir son deuil:

"C'est si dur de faire son deuil quand on n'a pas pu se dire au revoir..."

Ses parents lui manquent d'une manière lancinante:

"Mes parents me manquent, être orpheline est un abîme de solitude dont je ne guéris pas. ${ }^{\prime 6}$

Elle n'a pas réussi à faire son deuil parce que les bombardements allemands en 1945 ont complètement détruit tous les détails de sa vie:

"A Londres, chaque fois que je passais dans la rue où nous habitions, je ne reconnaissais plus rien, ni notre immeuble ni les petits magasins où je me rendais avec ma mère, car tout a disparu." ${ }^{7}$

Alice souffre secrètement, son inconscient commence à sonner le glas en émettant des signaux de perturbations graves. Chaque soir, elle est perturbée par des cauchemars terribles. Tous les événements traumatiques dont elle était victime se sont emmagasinés pendant de longues durées dans son inconscient qui se trouve dorénavant, incapable de s'exprimer. Il ressemble à un volcan endormi sur le point d'exploser:

"Il y avait des bruits de pas,poursuivit Alice,perdue dans ses pensées,des gens nous pourchassaient,(...).Je ne voyais qu'une main,elle m'entraînait dans une fuite terrifiante." ${ }^{\prime 8}$

Ainsi, la Première Guerre Mondiale et ses conséquences ont-elles arrachées Alice à ses parents biologiques et à sa terre natale. Elle en est sortie muette et amnésique. Cet événement a

\footnotetext{
${ }^{5}$ Lévy(Marc),op.cit..p. 268.

${ }^{6}$ Lévy(Marc),op.cit.,p.134.

${ }^{7}$ Levy,op.cit.,p.228.

${ }^{8}$ Levy,op.cit.,p. 132.
} 
fonctionné comme une bombe atomique qui a pulvérisé la vie d'une famille arménienne en paix. La Deuxième Guerre Mondiale vient pour achever la destruction psychique. A deux reprises, Alice se trouve privée de ses parents.

Dans la partie consacrée à la sémiotique de l'identité, nous allons démontrer comment Alice a pu surmonter tous ses désastres et réaliser sa propre catharsis.

\section{Événement social}

Alice part avec ses amis à la station balnéaire de Brighton. Par amusement, elle consulte une voyante.

Toute la sémiotique de l'événement requiert ici son importance. Par pur hasard,cette voyante était la sœur de la nourrice d'Alice. Elle l'a reconnue du premier coup et lui annonce:

"Il y a deux vies en toi,Alice. Celle que tu connais et une autre qui t'attend depuis longtemps.(pour découvrir tes origines,tu dois entreprendre un long voyage). Un voyage au cours duquel tu découvriras que rien de tout ce que tu croyais être n'était réalité". 9

A la grande surprise d'Alice, elle lui déclare avec une telle certitude que ses ancêtres sont natifs du Sud de l'Orient :

"Tu viens d'un empire qui n'existe plus, d'un très vieux pays distants de milliers de kilomètres. Le sang qui coule dans tes veines à pris sa source entre la mer Noire et la Caspienne". ${ }^{10}$

Les paroles de la voyante troublent Alice,surtout qu'elle souffre quotidiennement des cauchemars susmentionnés. Étant sûre de ses origines européennes, elle repousse ces prédictions. Cependant,les cauchemars ne cessent de perturber violemment ses nuits. Alice se résigne à l'idée de partir en Orient, non pas pour chercher ses origines, mais pour retrouver de nouvelles senteurs orientaux qui peuvent promouvoir sa création en parfum. Elle veut fuir Londres pour se régénérer:

\footnotetext{
${ }^{9}$ Levy,op.cit.,p. 45.

${ }^{10}$ Levy,op.cit.p. 46.
} 
"Ce ne sont pas seulement des parfums d'intérieur que je cherche,mais des parfums de lieux, ceux qui nous rappellent des instants qui nous ont marqués, des moments uniques et disparus. Saviez-vous que la mémoire olfactive est la seule qui ne se délite jamais. "${ }^{11}$

Elle nourrit l'espoir de ressusciter les moments uniques vécus avec ses parents adoptifs grâce à ses dons de parfumeur. Ici,apparaît l'amour de Marc Lévy pour le voyage.

Aidée financièrement par Mr. Dalry, son voisin, Alice part pour Istanbul pour retrouver sa joie de vivre et une promesse de régénération de ses capacités créatrices.

il paraît jusqu'à maintenant que les Événements traumatiques vécus par Anouche ou Alice ont joué le rôle de disjoncteur. Arracher Anouche à sa terre natale est une disjonction avec les origines et les lieux. Perdre la mémoire et la fonction oratoire est une disjonction avec ses propres atouts. Perdre ses parents biologiques est aussi une disjonction sur le plan familial. Les événements ont joué, dans ce cas, le rôle de déstabilisateur. Alice ressemble à une feuille d'arbre perdue et arrachée de ses branches à la suite d'un ouragan. Les actions qui se sont déroulées dans un espace donné: Istanbul et Londres, activent le sens de la disjonction entre le sujet d'état: Alice et ses objets de valeur: ses parents.

Nous allons voir si la sémiotique de l'espace qui entrera en jeu dès que les deux sujets d'État entreprennent leur voyage vers des espaces nouveaux,va remplir la fonction d'adjonction dans l'histoire d'Alice ou bien elle va alimenter la disjonction.

\section{II-Sémiotique de l'espace:}

Bernard Lamizet précise, dans sa Sémiotique de l'événement, que l'espace entre toujours en interaction dans la formation de la signification de l'événement:

${ }^{11}$ Levy,op.cit.p. 224. 
"La sémiotique de l'événement se fonde,(...) sur une sémiotique politique de la spatialité."12

La construction du sens lié à l'espace dans l'œuvre de Marc Lévy est très complexe.

\section{Espace disjoncteur:}

Les deux sujets d'état: Alice et M.Dalry partagent le même palier d'une ancienne maison victorienne. la verticalité est représentée par le bâtiment où ils vivent. Sur le plan horizontal,les deux appartements sont conjoints. Cependant,les deux zones topologiques sont contradictoires: la luminosité et la clarté de la chambre d'Alice sont en contradiction complète avec l'obscurité et la fermeture spatiale de la chambre de M.Dalry.

Une disjonction totale divise les deux voisins. Lorsque Alice reçoit ses amis, son voisin n'arrive pas,lui, à se reposer en calme:

"Je vivais ici bien avant vous, mademoiselle Pendlebury, mais depuis que vous vous êtes installée dans cet appartement,que j'espérais récupérer,ma vie est pour le moins perturbée et ma tranquilité n'est plus qu'un lointain souvenir." 13

L'espace occupé par Alice est convoité depuis longtemps par M.Daldry. C'est un peintre intéressé par la luminosité de la chambre d'Alice qui bénéficie d'une grande verrière éclairée:

"C'est pour cela que vous me battez froid depuis que je vis ici, M. Daldry? Parce que j'ai obtenu l'atelier que vous désiriez?."

Nous remarquons que la convoitise de l'espace d'Alice est la motivation qui va pousser $M$. Dalry à suggérer à Alice de financer son voyage. L'espace continue donc à jouer le rôle de disjoncteur entre Alice et son appartement.

\footnotetext{
${ }^{12}$ Lamizet, op.cit.,p.5.

${ }^{13}$ Lévy,op.cit..p. 10

${ }^{14}$ Lévy,op,cit.,p. 10
} 
Celle-ci demeure enfermée des jours entières, à court d'idées pour ses compositions florales. Elle souffre de solitude et de manque d'imagination productrice. Dans cet espace, elle est psychiquement bloquée:

"Je tourne en rond dans mon atelier,cela fait des mois que je n'ai plus rien créé, j'étouffe dans cette ville,dans cette vie. Je vais goûter l'air du large, m'enivrer de nouvelles senteurs et de paysages inconnus."

une autre fonction de l'espace apparaît avec la grisaille des cieux de Londres. Alice veut fuir la grisaille londonienne et partir vers la clarté de l'Orient. Elle affirme à M. Daldry:

"J'ai autant de plaisir que vous à quitter la grisaille londonienne. Partons aussi vite que possible. Je voudrais fuir l'hiver, Londres et mes habitudes, je voudrais que nous soyons déjà au jour du départ." ${ }^{\prime 16}$

De son côté, M. Daldry tourne en rond dans son appartement parce que son espace privé est pauvre en lumière nécessaire à son travail.

Les deux sujets d'état sont bloqués, autant sur le plan psychique que sur le plan créateur.

Nous devons ici préciser que leur cloisonnement respectif active le sens de l'étroitesse et de la fermeture spatiale. L'espace agit comme agent moteur et bloque également la production créatrice chez les deux protagonistes. C'est à dire que ce blocage psychique est en osmose avec le blocage spatial. Un état total de blocage dû a un espace hostile:

Fermeture spatiale + Solitude = manque de création.

Nous avons vu comment La fermeture spatiale alimente également l'obscurité psychique interne chez les deux sujets d'état. Un cercle vicieux dans lequel tourne les deux protagonistes:

\footnotetext{
${ }^{15}$ Lévy,op.cit..p. 101

${ }^{16}$ Lévy,op.cit.p.96.
} 


\section{Obscurité de l'espace topologique + Noirceur de l'espace psychique $=$ Disjonction de créativité.}

Si nous considérons que la zone topologique d'Alice est une zone A, celle de M. Daldry est une zone B, nous constatons que la zone $\mathrm{B}$, claire de l'extérieur, obscure de l'intérieur, renvoie à une résistance interne. La luminosité ne passe pas de l'espace externe:la rue, vers l'espace interne: l'esprit de M.Daldry. Il y a une résistance chez la zone B qui refuse et fuit la communication avec la zone A. Cette résistance d'ordre psychique sera analysée profondément dans la sémiotique de l'identité de M. Daldry.

La décision prise de partir pour la Turquie ensemble marque un pas progressif dans l'espace. Si le voyage d'Alice avec ses amis à Brighton a constitué un pas vers l'exploration spatiale, ce long voyage vers l'Orient marque un pas positif vers le mouvement. Ce mouvement directionnel jouera un rôle important dans la naissance d'une amitié entre les deux sujets.

Cela veut dire que chaque mouvement dans l'espace aura un rôle dans la conjonction des héros de l'histoire. Une conjonction amicale sera de naîtra.

\section{Voyage en Orient $=$ une trajectoire dans l'espace:}

Ce voyage vers Istanbul aura plusieurs points de visée focale, plusieurs objectifs. Alice part,en premier lieu, pour se ressourcer. L'Orient jouera ici le rôle d'un régénérateur grâce à la beauté des champs printaniers qui serviront de base à ses compositions florales.

"c'est un nouveau parfum que je suis venue chercher,et je l'imagine coloré, sensuel et en même temps léger". ${ }^{17}$

Notre héroïne tentera également de rechercher ses origines malgré son refus de l'idée d'être originaire de cette terre lointaine. Elle nourrit l'espoir que la découverte de cette terre mette un point final à ses nuits cauchemardesques.

${ }^{17}$ Lévy,op.cit.,p. 122. 
D'autre part, M.Daldry jouera le rôle d'un sujet opérateur à valeur modale riche ${ }^{18}$. Il aidera Alice dans tous les périples de son voyage et ne la quittera pas avant de s'assurer qu'elle est sur le point de réaliser ses rêves. Il retournera à Londres pour accomplir le deuxième volet de l'accord conclu. Il va profiter de la lumière de son appartement pour peindre.

Nous remarquons une progression de la zone spatiale A vers plus de lumière. Une lumière spirituelle et cognitive. Alice tentera de s'éclairer sur son passé et promouvoir son futur. Ce voyage constitue pour elle une transformation d'état. Elle bouge pour se transformer d'un sujet d'état à un sujet opérateur. Grâce au voyage, la zone $\mathrm{A}$ prend de la relève dès qu'elle commence le mouvement.

M.Dalry devient un agent de compétence modale de VOULOIR FAIRE. Épaulé d'un héritage formidable, il acquiert également la compétence de POUVOIR FAIRE. Il est maintenant,un agent de compétence modale riche de deux modalités: le VOULOIR FAIRE et le POUVOIR FAIRE.

M.Daldry essaye de rendre chaque périple de ce voyage similaire à un rêve ou à une valse. Marc Lévy use de beaucoup de fantaisie pour transformer ce voyage en un conte féerique. Les deux sujets actants dégustent avec volupté le goût parfumé d'un probable amour naissant.

Nous avons remarqué que les éléments spaciaux ont eu une représentativité importante. Une extension de la zone A, soit disant proximale vers une zone $\mathrm{A}^{* *}$ dite distale qui traduit la distance parcourue.

\section{Structure verticale de l'espace:}

Tout d'abord,M. Daldry a décidé de faire le voyage en avion, ce qui n'était pas de coutume en 1950. L'aviation coûtait cher. Entrer dans l'espace vertical de la hauteur a signifié une

\footnotetext{
${ }^{18}$ nous empruntons la terminologie de Greimas et Courtès employé dans le dictionnaire raisonné de la théorie du langage,hachette,1979, article"sujet" p. 264, article "objet" p. 265.
} 
montée dans la progression des sentiments entre les deux sujets. Chacun de sa part nie et refuse cette réalité.

Géographiquement parlant,Londres demeure au Nord du continent européen, c'est une localisation de hauteur en comparaison avec la Turquie dont la localisation est au sud du continent asiatique sur la carte géographique. Monter et descendre par avion résume un va et vient d'hésitation entre les deux cœurs.

M.Daldry avait organisé de passer voir une séance de l'opéra de Guilty puisqu'ils seront de passage à Vienne:

"une nuit à Vienne sans aller écouter une pièce de théâtre lyrique,c'était impossible."19

Lorsque l'avion a eu du retard,Alice et M. Dalry n'ont pas eu le temps de mettre leurs costumes formels pour un tel événement. Ils ont dû se faufiler par la porte de l'orchestre et regarder l'opéra en cachette. Ils se sont installés en HAUT du bâtiment. Cette localisation en hauteur peut signifier le bonheur créé grâce à la légèreté ${ }^{20}$. le bonheur de celui qui regarde d'en haut une ouverture d'horizon. le bonheur d'un oiseau qui sillonne le ciel à la recherche de son nid.

\section{Dimension cognitive du voyage:}

Le voyage commence à acquérir la valeur d'une compétence modale, celle de la découverte. Il remplit la fonction de réaliser une performance cognitive. Des qu'Alice a mis les pieds sur la terre turque, une étrange sensation d'avoir vécu en ces lieux l'envahit:

"C'est étrange,dit Alice, nous sommes bien loin de Londres, et cet endroit me semble familier". ${ }^{21}$

Chaque fois qu'elle se balade avec M.Dalry, elle est étonnée d'avoir la sensation de connaître ces lieux:

\footnotetext{
${ }^{19}$ Levy, op.cit..p.116.

${ }^{20} \mathrm{cf}$. Bachelard, la poétique de l'espace, puf,1957 premiere edition, 2012 dernière édition.

${ }^{21}$ Lévy,op.cit.,p. 125.
} 
"J'ai déjà vu cette maison, je connais cet endroit, murmura Alice.(...) Elle apparaissait dans chacun de mes cauchemars et se trouvait dans une ruelle au bout de laquelle un grand escalier descendait vers le bas de la ville." 22

ce processus étrange est connu sous le nom de cartes cognitives:

"Il s'agit de ce processus par lequel l'individu perçoit,emmagasine,rappelle,édite et communique les informations spatiales dont il dispose sur un environnement fréquenté". ${ }^{23}$

Il paraît qu'Alice avait réservé intacte dans ses structures cérébrales, malgré l'amnésie, des représentations du lieu où elle avait vécu à Istanbul. Les espaces vus dans les cauchemars se trouvent similaires aux lieux qu'elle visite. sa mémoire avait réservé le souvenir des rues et des ruelles de son enfance.

Les chercheurs en sciences cognitives considèrent que les informations et les souvenirs demeurent gravés dans la mémoire de l'individu:

"À partir de ces informations (emmagasinées), l'individu peut notamment concevoir des cartes cognitives, représentations de l'environnement qu'il créé mentalement.(...) $C^{\prime} e s t$ en ce sens qu'est considéré la cognition spatiale par les chercheurs en sciences cognitives et en neurosciences. ${ }^{24}$

Il paraît clair maintenant, pour quelle raison Alice s'attarde t'elle devant le petit magasin d'un cordonnier à Istanbul. Elle avait conservé intact dans sa mémoire l'odeur de cuir et de colle. Son père biologique était cordonnier:

"Alice s'attarda devant l'échoppe d'un cordonnier.(..)

-Alors pourquoi regardez-vous cet homme dans sa boutique depuis cinq bonnes minutes, sans rien dire?

${ }^{22}$ Lévy,op.cit, p. 132.

${ }^{23}$ Duroisin (Natacha),Demeuse (Marc),Bohbot (Véronique), Apprendre l'espace à l'école. cahiers pédagogiques, 527,2016,p. 48-49.

${ }^{24}$ ID, Ibidem. 
-Cela vous arrive que certaines choses anodines vous procurent une sensation d'apaisement sans que vous en compreniez la raison?"25

\section{Transmission de la compétence modale:}

Sur une terre étrangère,la compétence cognitive de M.Daldry se trouve freiné. Malgré ses compétences de VOULOIR FAIRE, il est stoppé par un non POUVOIR FAIRE. M. Daldry se trouve dans l'obligation de chercher un troisième actant qui possède cette modalité. Il a eu recours à un guide touristique nommé Can.

Il semble que Marc Lévy nous adresse un clin d'œil en donnant ce nom anglais dont le sens connotatif signifie POUVOIR FAIRE au guide qui jouera le rôle de conjonction entre Alice et sa terre natale. Celui-ci possède une formidable compétence modale et une pertinence de POUVOIR FAIRE grâce à son métier.

M. Can va agir comme catalyseur, toujours capable d'iradiquer toute confusion spatiale sur une terre nouvelle. Il empêche la dispersion des sujets actants dans les labyrinthes spatiaux mal connus.

Il aide Alice à retrouver son ancienne maison, à retrouver sa nourrice et conclure l'adjonction avec la terre d'origine. Il l'accompagne quotidiennement chercher dans les registres des écoles pour chercher les traces du passé. Il l'aide à travailler chez un parfumeur. Il a joué un rôle substantiel dans sa découverte des champs printaniers à Istanbul.

\section{Acquisition d'Alice de compétences modales:}

De ce mouvement enthousiaste dans l'espace nouveau, Alice commence à acquérir une certaine compétence modale de nature cognitive: elle visite les champs printaniers, se régale des divers parfums de fleurs, par conséquent,elle acquiert la compétence des lieux et des compositions florales.

${ }^{25}$ Levy,op.cit.,p. 205 . 
Elle commence également à acquérir une compétence modale de nature créative: elle se donne le temps pour apprendre et s'appliquer. Elle collabore quotidiennement avec un chimiste savant pour acquérir les secrets de ce travail artisanal et méticuleux avant d'atteindre cette phase de compétence créative.

\section{circularité du voyage:}

Marc Lévy a utilisé plusieurs marqueurs spatiaux pour désigner la circularité du voyage dans son roman. Les sujets partent de Londres vers Istanbul et y retournent. Les deux protagonistes avaient effectué ensemble un voyage de Londres à Brighton le jour de l'an, puis ils sont retournés vers leur point de départ. La circularité constitue en soi un signe d'accomplissement. Elle signifie l'accomplissement de la stabilité grâce à la conjonction entre les deux espaces.

La ligne du départ qui a tracé les premiers pas du mouvement se ferme sur elle même. la forme géométrique du cercle a pour fonction de réunir ce qui a été déjà séparé.

La première conjonction: Alice a vécu à Istanbul avant d'aller à Londres. Elle retourne à sa terre natale pour effectuer la conjonction avec les origines et opérer sa propre catharsis.

la deuxième conjonction: Alice découvre que M. Daldry l'aime profondément,elle prend la décision de le rejoindre pour toujours.

Alice a réalisé, de la sorte,une performance cognitive de grandes valeurs grâce à la circularité du voyage. Cette circularité fonctionne comme signe projetant le message de l'œuvre: se fixer un but,s'élancer vers sa réalisation, acquérir des compétences modales diverses et enfin retourner au même point de départ, vainqueur. Mais surtout, il faut bouger pour acquérir la transformation. À travers ce voyage, Alice a réussi tous ses objectifs: connaitre sa vraie histoire, se relier à sa terre natale,retrouver son frère et sa nourrice. Elle a également tenu sa promesse à M. Daldry, qui constitue à créer un parfum unique. Par cette conjonction, elle a également retrouver sa 
pleine liberté. Et lorsqu'elle rejoint l'amour de sa vie, le cercle de la conjonction se ferme après avoir rempli sa fonction de stabilité.

le cercle se ferme sur une conjonction de l'intimité entre les deux sujets actants. Enfin, une conjonction entre les Espaces traduit une conjonction entre les personnages.

Si la forme géographique du cercle a rempli cette fonction de conjonction, elle aura toute une autre signification dans la partie consacrée à l'étude de l'identité de M.Daldry.

En réalité, l'espace continue à agir sur la destinée des personnages. Nous allons découvrir comment? à travers l'analyse de la sémiotique de l'identité des deux sujets opérateurs.

\section{III-Sémiotique de l'identité d'Alice:}

Bernard Lamizet insiste sur l'interaction entre l'espace et la formation de l'identité:

"Un événement est,ce qui, survenu du dehors, du réel de la société, vient mettre à l'épreuve la sociabilité et la logique de l'appartenance et de l'identité. Ce sont,ainsi, deux sémiotiques qui sont articulées l'une à l'autre: celle de l'événement, qui est une sémiotique de l'espace et du temps, et celle de l'identité, qui est une sémiotique de la médiation entre sa dimension singulière et sa dimension collective." 26

Lamizet insiste donc,sur le fait que l'identité se fonde par la confrontation avec les expériences vécues et c'est exactement cela que Marc Lévy cherche à démontrer à travers l'histoire d'Alice et de M. Daldry.

Nous allons essayer d'analyser comment Alice a pu, malgré ses traumatismes se forger une identité particulière.

Pierre Bourdieu signale l'importance de la formation de ce qu'il appelle "l'habitus". Il souligne que cet "habitus" forme l'identité de la personne grâce aux dispositions qu'elle emmagasine tout au long de sa vie:

${ }^{26}$ Lamizer, op.cit.,p. 7 
"l'habitus est un système de dispositions durables acquis par l'individu au cours du processus de socialisation. Les dispositions sont des habitudes, des inclinations à percevoir,sentir,faire et penser, intériorisées par les individus du fait de leurs conditions objectives d'existence, et qui fonctionnent comme des principes inconscients d'action, de perception et de réflexion". ${ }^{27}$

Alice a été très sensible à la spécialisation de ses parents adoptifs. La vie en compagnie de deux pharmaciens qui effectuent des études de pharmacologie a donné à Alice l'envie de jouer avec les composants chimiques dans le but de former une substance utile. Ses parents adoptifs ont façonné, a leur insu, ses "SHÈMES DE PERCEPTION ET D'ACTION":

"On parlera d'ethos pour désigner les principes ou les valeurs à l'état pratique, la forme intériorisée et non consciente de la morale qui règle la conduite quotidienne:ce sont les schèmes en action, mais de manière inconsciente(...)." 28

Les parents adoptifs d'Alice ont réussi à lui inculquer d'une manière indirecte des "SHÈMES DE PERCEPTION" qui se sont transformés en "HABITUS PRIMAIRE":

"Parmi toutes les actions pédagogiques que nous subissons , les plus décisives sont les plus précoces,celles que nous avons subies au cours de notre enfance, et qui ont pour résultat de nous inculquer un habitus primaire. Celui-ci est constitué des dispositions les plus anciennement acquises et donc les plus durables. ${ }^{\prime 29}$

Lorsque la communication verbale a été bloquée à cause du traumatisme visuel subi lors de la persécution des parents, toutes les facultés sensorielles d'Alice ont été activées au maximum. Le sens de l'olfaction a été le plus aiguisé. Toute la

\footnotetext{
${ }^{27}$ Bonnewitz(Patrice), la sociologie de Pierre Bourdieu,PUF,Paris,1998,p. 62.

${ }^{28}$ Bonnewitz, op.cit..p. 63.

${ }^{29}$ Bonnewitz,op.cit.,p.64.
} 
communication avec le monde extérieur se passe dorénavant par le sens olfactif. Son langage consiste en un gigantesque vocabulaire d'odeurs. Elle avait le nez le plus fin au monde. Elle voyait par l'odorat. Alice était devenue un NEZ:

"Alice avait un don particulier. Ses facultés olfactives bien supérieures à la normale lui permettaient de distinguer la moindre senteur et de la mémoriser à jamais. Elle passait ses journées,(...) travaillant à combiner des molécules pour obtenir l'accord qui deviendrait peut-être un jour un parfum". ${ }^{30}$

Épaulée par ses parents adoptifs, elle commence à collecter la subtilité fine que contient l'arôme des fleurs.

"Mon père était pharmacien, moi je suis ce que l'on appelle un nez.J'essaie de créer des compositions de nouvelles fragrances". 31

Elle collecte les atomes du parfum avec une délectation exquise, comme si elle collecte les atomes dispersés de sa vie. le parfum devient,pour elle, l'expression des sentiments impossibles à communiquer par la langue oratoire.

La composition du parfum lui permet de recomposer une nouvelle existence adaptée avec sa nouvelle vie à Londres. Si les parents adoptifs lui ont inculqué un "HABITUS PRIMAIRE", Alice elle même, s'est forgée un "HABITUS SECONDAIRE":

"(...), sur l'habitus primaire se greffe, au fil du vécu de l'agent, des habitus secondaires." 32

De la sorte, elle se transforme pour la première fois de sa vie d'un sujet d'état à un sujet opérateur de sa propre vie.

En créant chaque composition,elle recrée un univers olfactif qui lui est propre. Elle bâtit pour elle même, un royaume olfactif. Un parfumeur ressemble en quelque sorte à un alchimiste qui transforme par magie Les éléments

\footnotetext{
${ }^{30}$ lévy,op.cit.,p.11.

${ }^{31}$ Levy,op.cit.p.51.

${ }^{32}$ Bonnewitz,op.cit.,p.64.
} 
premiers:arômes des fleurs en un Éden odorant. Le parfum,pour Alice, a joué le rôle d'un agent catalyseur qui a rafraîchi sa mémoire et a stimulé son imagination avant de rétablir son équilibre psychique.

L'objectif de son voyage était de créer un parfum nouveau, mais après avoir été éclairée sur son passé, elle s'est créée un nouvel objectif. Elle s'applique de nouveau à recréer un HABITUS SECONDAIRE pour retrouver son équilibre anéanti par les événements.

Elle s'applique dorénavant, à recréer toutes les odeurs de son enfance. Elle a voulu contenir toutes les fragrances de son enfance à l'intérieur d'un flacon de parfum, ce qui constitue pour elle, un art d'enfermer en soi, de garder pour soi le passé évanescent. Elle aspire retrouver les odeurs qui avaient ranimé tout son passé.

"Je voudrais pouvoir recréer des moments disparus, réveiller des lieux endormis ${ }^{\prime \prime 33}$

L'espace étroit d'un flacon de parfum constitue peut-être, du point de vue d'Alice, un mur solide et isolant contre la perte des souvenirs. Pour empêcher un passé évanescent de se disperser, elle cache ses souvenirs au sein d'un flacon de parfum. Comme si la consistance corporelle du flacon l'aidait à s'y réfugier. Comme si la bouteille solide lui fournissait un sentiment tactile de solidité en vue de se durcir contre des coups probables du sort.

Une réflexion s'impose ici, Si la fleur garde sa vitalité grâce à ses racines, Alice a retrouvé sa vitalité en nouant avec sa terre natale. Soulignons qu'il y a une analogie entre les racines des plantes collectées dans une bouteille de parfum et les racines anthropologiques de la personne.

Le parfum joue ici,le rôle de catalyseur de la mémoire. Il réveille le passé, le contient avant de l'incorporer dans le présent: un acte de création et de reconstruction:

${ }^{33}$ Levy,op.cit.,p. 227 
"Imaginez ce que cela représenterait de pouvoir retrouver l'odeur d'une pluie qui vous est familière?". ${ }^{34}$

Apres avoir pu s'accomplir psychiquement, Alice, réussit à composer le parfum de sa vie. Un parfum capable de ressusciter chaque instant vécu à Istanbul. Elle a travaillé assidûment et elle a gagné le pari. Elle a également tenu sa promesse avec M.Daldry :

"Daldry ôta le bouchon,ferma les yeux et respira le parfum. La note de tête était parfaite. Les yeux clos, Daldry fut transporté sous la frondaison des arbres de Judéee qui bordent le Bosphore. Il eut l'impression de remonter les ruelles(...),d'entendre la voix claire d'Alice(...). Il sentit l'odeur suave d'un accord de terre, de fleurs et de poussière,de l'eau fraîche qui coule sur la pierre usée des fontaines ${ }^{135}$

Avec cette création, Alice a réussi sa propre catharsis. Enfin, elle a possédé la modalisation du POUVOIR FAIRE et la compétence créatrice en se transformant d'un sujet d'état à un sujet opérateur de sa vie future. c'est lā seulement qu'elle goûte au bonheur après avoir pu chasser sa noirceur interne:

"Je suis heureuse, Daldry,vraiment heureuse. Je me sens plus libre que jamais, je crois même pouvoir affirmer que je n'ai jamais connu une telle liberté et qu'elle m'énivre". ${ }^{36}$

\section{IV-Sémiotique de l'identité de M. Daldry:}

Comme Alice a choisi de communiquer avec le monde à travers l'odorat, M. Daldry a choisi la peinture comme mode de communication avec le monde externe.

la problématique essentielle de l'identité de M. Daldry réside dans son besoin inassouvi de communication avec ses parents et surtout avec son père: "Mon père et moi ne sommes

\footnotetext{
${ }^{34}$ Lévy,op.cit.p.228.

${ }^{35}$ Lévy, op.cit.p. 300 .

${ }^{36}$ Id.,ibid.,p. 235 .
} 
d'accord sur rien,il trouve mon métier grotesque,moi le sien terriblement ennuyeux, bref,nous ne nous supportons pas. ${ }^{137}$

l'événement traumatique majeur qui a eu une forte résonance sur la vie psychique de M.Daldry était la traîtrise de son père. Celui-ci trahissait sa femme en toute impunité, ce qui a traumatisé très tôt du fils, trop attaché à sa mère.Touché profondément par la douleur secrète de sa mère, M.Daldry juge que son père,non pas seulement a trahi la vie conjugale,mais,il a également sali l'amour de sa mère: salissait". ${ }^{38}$

" (...) Mais c'était ma mère qu'il trompait et son amour qu'il

M.Daldry a été profondément affecté par la souffrance de sa mère:

"C'est la souffrance de ma mère que je ne supportais pas". ${ }^{39}$

\section{Conflit oedipien et sentiment de culpabilité:}

M.Daldry ressent un sentiment lourd de culpabilité parce qu'il se considérait incapable, ni de protéger sa mère de la douleur infligée, ni de lui porter remède. ce sentiment d'incapacité traduit une certaine confusion entre son identité et celle de son père.

Il paraît que M. Daldry n'avait pas investi le conflit œdipien parce que, quoique adulte, il s'identifiait toujours à son père: "Je suis le portrait de mon père". ${ }^{40}$

Etre enlisé dans la relation triangulaire du conflit œdipien n'a pas donné l'occasion à $M$. Daldry de s'investir psychiquement pour pouvoir aller vers l'Autre. Il en a résulté une peur bleue d'investir son amour pour Alice. Il a toujours fui sa compagnie pour ne pas permettre à ce sentiment de prendre plus de consistance. Il décide de demeurer en permanence, loin pour demeurer chaste. Son identification

\footnotetext{
${ }^{37}$ Levy, Op.Cit.,p.32.

${ }^{38}$ Lévy, op.cit., p. 204.

${ }^{39}$ Id.,ibid.,p. 203.

${ }^{40}$ Id, ibid.,p. 201
} 
maladive à son père ne lui a pas permis de casser l'écorce de l'œdipe. Chaque fois qu'il essaye d'aller vers l'Autre, le retour du refoulé s'installe et les mécanismes de défense battent leur plein. Ces mécanismes de défense consistait à fuir l'amour (d'Alice) à tout prix:

"Si je tombais amoureux d'une femme comme elle, reprit-il, la seule preuve d'amour que je pourrais lui offrir serait de m'éloigner le plus loin possible, dussé-je aller au bout du monde". ${ }^{41}$

C'est à dire que ses mécanismes de défense mis en place ont choisi de s'éloigner de la personne qu'il aimerait:

"Dans une histoire d'amour, il faut savoir partir avant qu'il soit trop tard;(...). ${ }^{42}$

Secoué intérieurement par ces violents sentiments de culpabilité, il est proie à une névrose obsessionnelle:

"Le sentiment de culpabilité (inconscient envers la douleur de sa mère) est organisateur de la névrose obsessionnelle.(...) Le "Surmoi" est très violent, très contraignant, il fait des reproches au "moi" et bien qu'il se sache innocent, il se sent coupable: contrainte du "moi" à obeïr au "Surmoi" jusqu'à épuisement à travers les symptômes et les défenses mis en place." 43

\section{Névrose obsessionnelle de M.Daldry:}

De ce qui précède, nous pouvons déduire que $M$. Daldry fut atteint d'une névrose obsessionnelle qui bloque son investissement psychique de pouvoir aller vers l'autre. Du point de vue étymologique, l'obsession veut dire assiéger ou être assiéger:

"Obsession correspond à une idée obsédante, intrusion dans la conscience d'une pensée qui assiège la conscience et

\footnotetext{
${ }^{41}$ levy,op.cit.,p.200.

${ }^{42}$ Id., Ibid.,p. 201.

${ }^{43}$ Sans nom d'auteur, psychopathologie générale de l'adulte: sémiologie, nosographie et développement psychique. http: // membres multimania. p. 10.
} 
dont le sujet n'arrive pas à se débarrasser. Il reconnait que cette idée obsédante est ridicule, voire morbide (maladive) mais il ne peut s'en empêcher. Toute la volonté qu'il développe est inefficace. ${ }^{\prime \prime 4}$

Alors, lorsque M. Daldry est tombé amoureux d'Alice, cet amour a activé ses moyens de défense. Pour amoindrir la perturbation de l'inconscient, il préfère l'éloignement. S'approcher de la personne qu'on aime dans ce cas, constitue une réactivation de tous les maux ensevelis dans le tréfonds de l'inconscient:

"L'omniprésence de défense conduit à une importante ambivalence ( entre désir et interdit)". ${ }^{45}$

Tant que ces mécanismes de défenses fonctionnent, $M$. Daldry fuit le contact avec Alice: "Daldry était tétanisé" ${ }^{46}$ de la toucher:

"La phobie du contact est présente dans les descriptions freudiennes (tabou du toucher) et prend sens comme la mesure qui évite de se rapprocher de l'objet du désir(...). L'obssession évite le conflit oedipien". ${ }^{47}$

Ainsi, parait-il, clair maintenant que M. Daldry avait souffert d'une mauvaise relation avec ses parents, surtout avec son père, ce qui a laissé de graves conséquences sur sa vie future. M. Daldry avait entamé la logique de l'amour. La logique de tout amour implique un VOULOIR ETRE PROCHE, c'est à dire conjoint à la personne aimée. Or, ce nest pas le cas chez M. Daldry qui fuit la promiscuité: "Je déteste la promiscuitén

permettez-nous d'expliquer cette relation étrange à travers le carré sémiotique de GREIMAS:

\footnotetext{
${ }^{44}$ Sans nom d'auteur, op.cit..p. 8

${ }^{45} \mathrm{Id}$, ibid.,p. 10.

${ }^{46}$ Levy,Op.,Cit.,p.120.

${ }^{47}$ Sans nom d'auteur, Op.Cit.,p.8.

${ }^{48}$ Levy,Op.,Cit., p. 200.
} 
Bulletin de La Faculté des Lettres, vol. (48), ${ }^{\circ}$ (1), Juillet 2018

S1

VOULOIR ETRE CONJOINT

= PROMISCUITÉ

Š2

NE PAS VOULOIR

être disjoint $=$

cohabitation $=$

vivre proche $=$

chacun dispose de sa liberté

et de son espace propre .
S2

VOULOIR ETRE DISJOINT

$=$ FUITE

Š1

NE PAS VOULOIR ETRE

conjoint $=$

refuser le partage $=$

refuser la promiscuité

La logique de l'amour implique que le sujet agent veuille chercher la promiscuité avec son objet d'amour. Aimer,c'est vouloir adhérer,être proche,vivre auprès de la personne aimée $====\gg 》 \gg S$ 1. Pourtant, le sujet actant M. Daldry ne veut pas être proche.Il fuit l'amour,et refuse l'adhérence $======》$

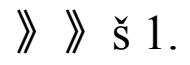

L'inconscient de M. Daldry fuit son objet d'amour, l'aide à voyager pour quitter Londres et essaye de la convaincre d'aller chercher l'homme qui comptera le plus dans sa vie. C'est un vouloir être disjoint qui a des raisons psychiques susmentionnés $=====》\rangle \gg \mathrm{S} 2$.

M.Daldry oscille donc, entre VOULOIR ÊTRE PROCHE (S 1), et VOULOIR ETRE LOIN (S 2). Il ne veut pas être totalement disjoint. Il cherche une sorte de cohabitation: vivre 
près de son objet d'amour à condition que chacun dispose de sa liberté $=====》\rangle 》($ s 2$)$.

NE PAS VOULOIR ÊTRE CONJOINT (ک̌́1) ou VOULOIR ÊTRE DISJOINT (S2) traduit la crise passionnelle dont souffre M.Daldry. La crise passionnelle s'alimente volontiers de l'action la plus anodine: le toucher des mains. Lorsque Alice touche les mains de son ami à Istanbul, elle enclenche le processus de défense:

"Alice prit la main de Daldry qui frôlait la sienne". 49

Le lendemain, Daldry part définitivement pour Londres et laisse Alice à Istanbul. Il quitte sa bien aimée, retourne à Londres de peur de s'enliser davantage dans une relation charnelle. M.Daldry quitte Istanbul pour Londres afin de se consacrer à la peinture sous la verrière éclairée de la chambre d'Alice. On va essayer d'analyser l'apport positif que va exercer son application à la peinture pour réaliser son chef d'œuvre personnel. Par quel moyen, se transformera l'état de M. Daldry, d'un sujet d'état à un sujet opérateur de sa propre vie?

\section{Sémiotique de la peinture:}

M.Daldry était peintre, sensible à la peinture des carrefours. La logique spatiale de M. Daldry s'exprimait à travers la figure géométrique du cercle. Il ne peint que des carrefours.

Le carrefour est un espace circulaire qui ne permet ni le mouvement directionnel ni l'extension dans l'espace. Le cercle brise l'ouverture spatiale parce qu'il se ferme sur lui-même. M.Daldry, incapable de communiquer avec ses parents, était emprisonné dans le cercle de ses névroses obsessionnelles. Un état d'incommunicabilité ne trouve son expression que dans la peinture de cercles:

"Vous n'imaginez pas à quel point la vie à un carrefour est riche de mille détails.(...) Un carrefour est un endroit passionnant. ${ }^{\prime 50}$

\footnotetext{
${ }^{49}$ Levy, Op.,cit., p. 229.

${ }^{50}$ Levy, Op. cit., p. 33.
} 
On peut dire que ce point zéro du mouvement ou de direction (qui tourne en rond) met l'accent sur un blocage psychique.

Le peintre tourne en rond dans son espace psychique et géographique à la fois. Il tourne, étouffé par ses problèmes et se trouve incapable de résoudre ses complexes. Au lieu de jouir de la présence d'Alice et arpenter des espaces nouveaux, M.Daldry choisit de se cloisonner entre 4 murs pour peindre des carrefours. Ce cloisonnement active le sens du repliement sur soi. C'est une réduction dans le mouvement spatial qui active sa solitude psychique. Elle alimente également l'obscurité psychique interne de notre sujet d'état:M.Daldry. En fuyant son amour,M. Daldry cherche à réserver son intimité propre. Ce fut une disjonction avec l'amour en profit d'une conjonction avec l'intimité. L'espace remplit,pour lui,la fonction d'un abri:

"Du point de vue thymique, la configuration de l'abri suppose une relation fiduciaire $d u$ sujet à l'espace: LA QUIÉTUDE" ${ }^{\prime 51}$.

Lorsqu'il a conclu l'accord avec Alice pour peindre sous sa verrière, il cherchait l'énergie incluse dans la matière de la LUMIÈRE:

"L'éclairage est un acte, c'est la mise en oeuvre de la lumière pour remplir une fonction. C'est donc l'éclairage qui est porteur d'une signification, c'est lui le signe et non la lumière." $" 52$

Nous pensons que la peinture est un outil communicationnel par excellence. En cherchant plus de luminosité, M.Daldry voulait fuir en même temps sa noirceur interne. Et puisque sa névrose lui dicte l'éloignement de son

\footnotetext{
${ }^{51}$ Roelens(nathalie), Sémiotique urbaine et géocentrique, presses universitaires de Liège,2015,p.176.

${ }^{52}$ Ezrati (Jean-Jacques), " pour une sémiotique de l'éclairage d'exposition" in Lux,n. 218- mai/juin 2002,p.27.
} 
amour, son inconscient a cherché une sorte de compensation en voulant acquérir l'espace où vit la bien-aimée. Pour demeurer en état de conjonction, Daldry a voulu se procurer son espace intime pour s'y abriter contre ses peurs. cette chambre équivaut à un abri qui réalise un confort. Pénétrer l'intimité spatiale d'Alice le dispense de vivre avec elle dans un état d'intimité charnelle. cet état de compensation le rend en sécurité, calme son inconscient dévoré par la névrose obsessionnelle.

L'éclairage que procure la grande verrière de la chambre d'Alice active le processus dynamique de sa progression psychique:

" La lumière qui tombe de votre verrière est presque une illumination pour moi, je n'ai jamais aussi bien travaillé". ${ }^{53}$

En prenant place dans la chambre d'Alice, la luminosité commence à envahir l'inconscient de M.Daldry, la zone B par extension du sens, pour disperser son obscurité psychique. La luminosité envahit lentement la zone psychique $\mathrm{B}$ à travers son extrémité verticale. A mesure que la relation amicale se solidifie entre Alice et M. Daldry, la luminosité accroît et envahit le cour de celui-ci. Une probable ouverture ou une guérison prochaine s'annonce. A plusieurs reprises, M. Daldry a fui la présence d'Alice. Il l'a quitté à Istanbul des qu'il a senti que son amour grandit. Lorsqu'elle lui a annoncé qu'elle le rejoindrait à Londres, il a présenté des prétextes qu'il n'y serait plus à cette date. Après qu'Alice a découvert la réalité de son amour, et après l'avoir affronté, il a dû finalement avouer cet amour. Lorsque Alice lui pose la question: depuis quand il l'aime? Il lui répond:

"Depuis le premier jour où vous êtes entrée dans cette maison, depuis la première fois où je vous ai vue monter cet escalier et le trouble n'a cessé d'empirer. ${ }^{154}$

\footnotetext{
${ }^{53}$ Levy, Op.Cit.,p. 246.

${ }^{54}$ Levy,op.cit.p. 294 .
} 
ce n'est que plus tard qu'il lui avoue ses angoisses et comment ses mécanismes de défense n'ont pas cessé de battre leur plein:

"De toute ma vie,je n'avais jamais craint quelqu'un comme je vous ai crainte. L'idée de vous aimer autant me faisait plus que jamais redouter de ressembler à mon père,(...)" ${ }^{155}$.

Ainsi, pouvons nous déduire que le $\operatorname{topos}^{56}$ qui est une parcelle d'espace ayant joué une fonction, celle de guérir un inconscient traumatisé était le catalyseur qui a mené à la guérison de M. Daldry. Il avoue:

"(...) vous m'avez frôlé l'âme et vous m'avez changé, comment vous pardonner d'avoir fait naître en moi l'envie d'aimer et d'être aimé? D'une façon très étrange, vous avez fait de moi un meilleur peintre, peut-être même un meilleur homme". 57

Le Topos est représenté par un studio à verrière lumineuse qui illumine l'obscurité psychique de la zone B.

Ainsi, la fonction du Topos provient du fait de l'action qu'a exercé cette parcelle d'espace sur la vie psychique du sujet d'état. Le Topos a joué dans le cas de M. Daldry la fonction du sujet opérateur qui a mené à la guérison. M.Daldry n'a pas prodigué l'effort nécessaire pour la transformation de son état. Il n'a pas pu acquérir,à l'encontre d'Alice, des compétences cognitives. Le Topos a fonctionné pour lui. Ici, le composant spatial supplante l'action humaine et sert à son rétablissement. L'homme est dominé par l'Espace, soumis à sa force. L'adjonction entre la composante : Lumière (l'éclairage) au rôle

\footnotetext{
${ }^{55}$ Id., ibidem.

56" Le Topos est une portion d'espace, découpée dans le continuum spacial, identifiable par ce qu'une action s'y accomplit. En d'autres termes, cest la portion discrète d'espace correspondant à l'accomplissement d'une action particuliěre". Glassner (J.J.), Ecrire à sumer,Paris, seuil,2000,p.10.

${ }^{57}$ Levy,op.cit.p. 294.
} 
du Topos aident M. Daldry à trouvé son objet de valeur final: la peinture vivace et illuminée du carrefour d'Istanbul.

Eclairage (synonyme d'illumination) + Topos (melting pot du génie longtemps nié de M. Daldry) = un meilleur peintre ouvert à la vie, à l'amour et surtout à la liberté.

\section{conclusion:}

A la fin de notre étude, nous avons constaté que Marc Lévy a choisi deux métiers qui font appel à la communication. Peindre, c'est transmettre sa propre vision du monde à travers le dessin. C'est un besoin de vouloir aller vers l'autre,lui communiquer ses idées. Peindre peut aussi être l'expression d'une liberté assiégée.

La parfumerie est également un genre d'échange et de communication. Composer le parfum ou s'en réjouir fait également appel à un genre de communication subtile. un interéchange entre l'humain et le floral.

Nos deux sujets avaient réussi leurs chemins vers l'acquisition du bonheur, chacun à sa manière.

Nous pensons que Marc Lévy,en dressant des obstacles devant ses protagonistes, a voulu,peut-être, savoir ce qu'ils feraient de leurs libertés réciproques.

Malgré l'atrocité des Evénements rencontrés par les protagonistes, malgré les blocages psychiques dressés devant l'acquisition de leurs libertés, ils ont eu le courage de les dépasser.

A travers une histoire sensuelle, Marc Lévy nous invite à réfléchir, chacun de sa part, sur le chemin à prendre. Il nous invite à penser quelles compétences doit-on acquérir pour dénouer les complexes et surmonter le pire. Il nous invite à aimer la vie. Il veut nous montrer comment acquérir nos objets de valeur dont le plus important est la liberté.

Lorsque Bernard Lamizet, à travers sa Sémiotique de l'événement, a voulu étudier l'impact des événements sur la vie des personnages, il a pris soin de la dimension spatiale et 
temporelle dans la formation de l'identité. Alors, le corpus de Marc Lévy nous a offert l'occasion d'analyser comment l'identité des protagonistes a été formée malgré les événements durs.

Nous avons découvert que l'espace et le temps peuvent aider avec beaucoup de patience a l'éclosion d'une identité ouverte,équilibrée, libre, et surtout capable de communiquer sainement. La liberté de choisir le chemin a prendre, l'effort déployé pour s'épanouir, l'identité reformulée sont les maîtres mots de l'étrange voyage de $M$. Daldry. Une identité reformulée, mais surtout polie par la gravité des obstacles surmontés semble être le message de l'œuvre.

\section{Bibliographie:}

- Le corpus: L'étrange voyage de M. Daldry, Paris,Laffont,2011.

\section{ouvrages critiques:}

- Bachelard, la poétique de l'espace, puf,1957 premiere edition, 2012 dernière édition.

- Bertrand(jeanpierre),Provenzano(François),Stiénon(Valerie), Litterature et sémiotique: histoire et épistémologie, presses universitaires de Liège, 2015.

- Bonnewitz(Patrice), la sociologie de Pierre Bourdieu,PUF,Paris, 1998,

- Charaudeau(Patrick), la médiation des controverses scientifiques, CNRS, Laboratoire Communication et politique,paris, 2015.

- Duroisin (Natacha),Demeuse (Marc),Bohbot (Véronique), Apprendre l'espace à l'école. cahiers pédagogiques, 527,2016.

- Ezrati (Jean-Jacques), " pour une sémiotique de l'éclairage d'exposition" in Lux,n. 218- mai/juin 2002,p.27.

- Glassner (J.J.), Ecrire à sumer,Paris, seuil,2000. 
- Lamizet(Bernard), la sémiotique de l'événement: une sémiotique de l'espace et du temps. 2011<halshs00604452>.

- Mucchielli(A), théorie des processus de la communication,Paris,Armand colin,1998.

- Mucchielli(A), Manuel de sémiotique situationnelle pour linterprétation des conduites et des communications. Montpellier: le moine copiste,2008.

- Roelens(nathalie), Sémiotique urbaine et géocentrique, presses universitaires de Liège, 2015,p.176.

- Sans nom d'auteur, psychopathologie générale de l'adulte: sémiologie, nosographie et développement psychique. http: // membres multimania.

\section{Actes de colloque}

- Navarette(Pierre-Antoine),Evolution du parcours generatif:vers un remaniement des structures spatiales, Congrès Mondial de Linguistique française-CMLF 2014.

\section{Articles de journaux:}

- Charaudeau(Patrick),Une analyse sémiolinguistique du discours.in Langages,29eme année, ${ }^{\circ}$ 117,1995,pp.96-111.

- Cosnier(Jacques),Vaysse(Jocelyne), Sémiotique des gestes communicatifs, in Nouveaux actes sémiotiques,52,7-28, 1997

- Duchatel(A), Marc lévy: bienheureux les doux ,in Entre les lignes, érudit,2007,pp.3(2),13.

- Hammad(Manar), La sémiotisation de l'espace, Esquisse d'une manière de faire, in Actes Sémiotiques, $\mathrm{n}^{\circ} 116$.

- IBO(Lydie), Approche comparative de la narratologie et de la sémiotique narrative, in Revue du Cames-nouvelle sérieB, volume $008 \mathrm{n}^{\circ} 2,2007$,(1er semestre).

- Jürges(Christina), Lieux imaginaires et espaces sémiotiques, in Figura,n²7,2011,pp.105-120. 
- Pullman(Micheal), Habitus et classes:les pistes ouvertes par la sociologie de Pierre Bourdieu, in Cahiers CEFRES numero 29,2003, pp.9.

- Ringham (Felizitas), Le parfum de l'écriture, in La Chouette,2000, pp.35-40.

- Taverna(Licia), L'espace comme dispositif sémiotique dans la mère sauvage de Maupassant in Synergies $\mathrm{n}^{\circ}$ 5,2008,pp.153-183.

- Cours de sémiologie: Benavides(Thierry), Sémiologie psychomotrice de l'enfant, Université Pierre et Marie Curie, 2002-2003.

\section{Thèses:}

- Gorelik(Polina), L'expression de l'odeur dans l'interaction du verbal et du visuel, thèse de doctorat sous la direction de Fontanille (Jacques), Université de limoges,Département Sciences du langage, 2008.

\section{Dictionnaire:}

- Greimas et Courtès employé dans le dictionnaire raisonné de la théorie du langage,hachette,1979, article"sujet" p. 264, article "objet" p. 265.

\section{Webiographie:}

- URL:http://id.erudit.org/iderudit/1017363ar. document téléchargé le 26 nov. 2016.

- URL:https//halshs.archives-ouvertes.fr/halshs00604452.www.erudit.org. 